\title{
Hydroxysafflor Yellow A mitigated myocardial ischemia/reperfusion injury by inhibiting the activation of the JAK2/STAT1 pathway
}

\author{
DONGLAI ZHOU*, TINGTING DING*, BIN NI, YUNYAN JING and SHANXIN LIU
}

Department of Cardiology, The Affiliated Hospital of Hangzhou Normal University, Hangzhou, Zhejiang 310015, P.R. China

Received June 20, 2018; Accepted April 17, 2019

DOI: $10.3892 /$ ijmm.2019.4230

\begin{abstract}
Hydroxysafflor Yellow A (HSYA) may reduce ischemia/reperfusion (I/R) injury. However, the underlying molecular mechanisms remain unclear. The present study explored the effect and the mechanisms of HSYA on myocardial injury in vivo and in vitro. Myocardial infarct size was assessed by Evans blue/2,3,5-triphenyltetrazoliumchloride staining. Levels of cardiac troponin I (cTnI), interleukin-6 (IL-6), lactate dehydrogenase (LDH), superoxide dismutase (SOD) and malondialdehyde (MDA) were measured using commercial kits. Alteration of mitochondrial membrane potential (MMP) and reactive oxygen species (ROS) generation was determined by fluorescent signals. Apoptosis was detected by terminal deoxynucleotidyl-transferase-mediated dUTP nick-end labeling staining, flow cytometry assay and caspase-3 activity. Expression levels of the apoptosis-associated proteins were detected by reverse transcription quantitative polymerase chain reaction and western blot analysis. In vivo, animals treated with HSYA presented less severe myocardial injury and decreased janus kinase 2 (JAK2)/signal transducer and activator of transcription 1 (STAT1) activity, improved antioxidant capacity and decreased apoptosis. In vitro, compared with the hypoxia (H)/reoxygenation (R) + HSYA group, AG490 and S1491 treatment decreased the releases of cTnI, IL-6 and LDH and enhanced the resistance to oxidative stress by maintaining MMP and decreasing ROS generation. In addition, AG490 and S1491 were also identified to alleviate the H/R-induced apoptosis by inhibiting caspase 3 activity and modulating the expression levels of cleaved caspase-3, tumor necrosis factor receptor superfamily member 6 (Fas), Fas ligand,
\end{abstract}

Correspondence to: Dr Shanxin Liu, Department of Cardiology, The Affiliated Hospital of Hangzhou Normal University, 126 Wenzhou Road, Hangzhou, Zhejiang 310015, P.R. China E-mail: shanxl_liusx@163.com

*Contributed equally

Key words: hydroxysafflor Yellow A, janus kinase 2/signal transducer and activator of transcription 1, myocardial ischemia/reperfusion injury
B-cell lymphoma 2 (Bcl-2) and Bcl-2-associated X protein. These data suggested that inactivation of the JAK2/STAT1 pathway strengthened the HSYA-induced protective effect in $\mathrm{H} / \mathrm{R}$-induced myocardial injury. In conclusion, the treatment of HSYA was effective in decreasing IR-induced myocardial injury, and this may be largely dependent on the JAK2/STAT1 pathway. Therefore, the present study provided a potential strategy to prevent myocardial I/R injury.

\section{Introduction}

Myocardial ischemia and reperfusion (I/R) injury is a primary risk factor for causing myocardial infarctions (MI), which is the most common cause of mortality in developed countries (1-3). It has been estimated that $\sim 15.9$ million individuals worldwide suffered from MI in 2015 (4). Although reperfusion therapy has been demonstrated to be able to restore impaired cardiac function and to mitigate the infarct size following ischemic events, the additional tissue injury caused by reperfusion is almost inevitable (5).

Multiple mechanisms are hypothesized to be involved in I/R injury (1). The inflammatory response to $I / R$ is common in myocardial I/R injury (6). Various cytokines will be released during the inflammation reactions (7). Reactive oxygen species (ROS) generation also occurs in the myocardium; however, if ROS cannot be cleared promptly, intracellular oxidative stress will be initiated, finally leading to cell apoptosis. Cellular apoptosis is known to be triggered shortly following MI, and it is evidently increased during reperfusion $(8,9)$. Apoptosis has been recognized as a crucial factor in the progression of $\mathrm{I} / \mathrm{R}$ injury in cultured myocardial cells $(10,11)$. Therefore, a decrease in inflammation, ROS generation and/or apoptosis may be therapeutic targets against I/R injury. Various apoptosis pathways, including the mitochondrial pathway and the tumor necrosis factor receptor superfamily member 6 (Fas)/Fas ligand (FasL) death receptor-mediated pathway, have been demonstrated to be essential for the apoptosis observed in the myocardium following I/R (11-14). Therefore, depressing apoptotic signaling may be a promising strategy to decrease I/R injury.

Carthamus tinctorius $L$. has been applied extensively in the treatment of cerebrovascular and cardiovascular diseases. Hydroxysafflor Yellow A (HSYA) is the primary active component of this compound, commonly used in Chinese medicine. 
The antioxidant effect of HSYA has been validated (15), and it has been demonstrated that HSYA may improve I/R injury by decreasing oxidative stress in brain tissue (16). Nevertheless, the mechanisms of HSYA-mediated protection from I/R injury are not completely understood.

The Janus kinase (JAK)/signal transducers and activators of transcription (STAT) pathway is able to modulate stress-responsive gene expression $(17,18)$. The phosphorylation of STATs mediated by JAK triggers alteration of gene transcription $(19,20)$. In addition, STATs have been demonstrated to regulate apoptosis in multiple cell types (21). Previous studies identified that STATs may modulate the opening of mitochondrial permeability transition pores $(22,23)$. In addition, activation of JAK/STAT signaling was identified in the development of renal I/R injury, in which the release of inflammatory promotion factors was enhanced (24). Previous data have also demonstrated that STAT1 is able to induce the expression levels of pro-apoptotic genes including Fas and FasL (25), and to repress the expression levels of anti-apoptotic gene including B-cell lymphoma 2 (Bcl-2) (26).

The present study was undertaken to examine the potential role of HSYA in myocardial I/R injury in vivo and in vitro. Furthermore, considering the crucial role of JAK/STAT1 in tissue protection, the potential role of this signaling pathway in HSYA-mediated protection against myocardial I/R injury was also examined.

\section{Materials and methods}

Chemical preparation. HSYA powder (95-99.0\% purity, determined by high performance liquid chromatography) was obtained from Shanghai Yuanye Biotechnology Co., Ltd. (Shanghai, China). Sodium pentobarbital was obtained from Sigma-Aldrich; Merck KGaA (Darmstadt, Germany). Cardiac troponin I (cTnI; cat. no. m1059498) and interleukin-6 (IL-6; cat. no. ml064292) ELISA kits were obtained from Shanghai Enzyme-linked Biotechnology Co., Ltd. (Shanghai, China). The superoxide dismutase (SOD; cat. no. S0101), lactate dehydrogenase (LDH; cat. no. C0016) and malondialdehyde (MDA; cat. no. S0131) kits were purchased from Beyotime Institute of Biotechnology (Haimen, China). Dulbecco's modified Eagle's medium (DMEM) and other cell culture reagents were purchased from Gibco; Thermo Fisher Scientific, Inc. (Waltham, MA, USA). Evans blue and 2,3,5-triphenyltetrazolium chloride (TTC) were obtained from Sigma-Aldrich; Merck KGaA. The specific inhibitors of JAK2 and STAT1 (named AG490 and S1491, respectively) were purchased from MedChemExpress (Monmouth Junction, NJ, USA). The antibodies used were purchased from Abcam (Cambridge, MA, USA) and Cell Signaling Technology, Inc. (CST, Inc.; Danvers, MA, USA), and were as follows: Anti-cleaved caspase (cat. no. ab49822; dilution, 1:500; Abcam), anti-Fas (cat. no. ab82419; dilution, 1:1,000; Abcam), anti-FasL (cat. no. ab15285; dilution, 1:500; Abcam), anti-Bcl-2 (cat. no. ab59348; dilution, 1:1,000; Abcam), anti-Bcl-2-associated X protein (Bax; cat. no. ab32503; 1:3,000; Abcam), anti- phosphorylated (p)-JAK2 (cat. no. 4406; dilution, 1:1,000; CST, Inc.), anti-p-STAT1 (cat. no. 7649; dilution, 1:1,000; CST, Inc.), anti- $\beta$-actin (cat. no. ab8226; dilution, 1:5,000; Abcam), anti-GAPDH (cat. no. ab9385; dilution, 1:1,000; Abcam) and horseradish peroxidase (HRP)-conjugated secondary antibodies (cat. no. ab205718; dilution, 1:5,000; Abcam).

$I / R$ model in vivo. The present study was performed, according to the Guide for the Care and Use of Laboratory Animals, 8th Edition (27) and approved by the Animal Subjects Committee of the Affiliated Hospital of Hangzhou Normal University (Hangzhou, China). The experiments were performed on 40 healthy adult male Sprague-Dawley rats (weighing $250 \pm 20 \mathrm{~g} ; 2$ months old) obtained from Guangdong Medical Laboratory Animal Center (Foshan, China). The animals were housed in a pathogen-free environment and maintained following standard laboratory animal feeding protocols. The animals had ad libitum access to food and water in a light/dark cycle (12/12 h). The animals were given 2 weeks to acclimate prior to the experiments. The local myocardial I/R model was constructed as follows: The animals were anesthetized by intraperitoneal administration of sodium pentobarbital $(60 \mathrm{mg} / \mathrm{kg})$; ligation of the anterior descending thoracic branch of the coronary artery was performed for $30 \mathrm{~min}$ prior to $2 \mathrm{~h}$ of perfusion. The animals were randomly divided into four groups, which were: Control (control), which comprised rats without I/R treatment; the Sham operated group (sham), in which open heart surgery was performed but the anterior descending branch of the coronary artery was not ligated; the $I / R$ group (I/R), which included rats that underwent $I / R$ treatment; and the $\mathrm{I} / \mathrm{R}+\mathrm{HSYA}$ group $(\mathrm{I} / \mathrm{R}+\mathrm{H})$, which included rats that underwent $\mathrm{I} / \mathrm{R}$ treatment, but $5 \mathrm{mg} / \mathrm{kg}$ HSYA was intraperitoneally injected $30 \mathrm{~min}$ prior to ischemia. As previously described and following our preliminary experiments, concentration of HSYA was determined $(28,29)$. Following perfusion, the anesthetized animals were prepared for subsequent experiments. Finally, the animals were sacrificed by intraperitoneal injection of sodium pentobarbital $(200 \mathrm{mg} / \mathrm{kg})$. Mortality was verified by lack of visible signs of respiration and measurable heartbeat.

$I / R$ model in vitro. H9c2 cells (American Type Culture Collection, Manassas, VA, USA) were cultured in DMEM medium containing fetal bovine serum (10\%) and streptomycin/penicillin (1\%) in an incubator with $5 \% \mathrm{CO}_{2}$. The cells were seeded at a density of $2.5 \times 10^{3}$ cell/well. The cells were randomly grouped as follows: i) Control group (control); ii) the hypoxia/reoxygenation group (H/R); iii) the $\mathrm{H} / \mathrm{R}+20 \mu \mathrm{M}$ HSYA group (H/R $+\mathrm{H})$; iv) the $\mathrm{H} / \mathrm{R}+10 \mu \mathrm{M}$ AG490 group $(\mathrm{H} / \mathrm{R}+\mathrm{A}) ; \mathrm{v})$ the $\mathrm{H} / \mathrm{R}+10 \mu \mathrm{M}$ AG490 $+20 \mu \mathrm{M}$ HSYA $(\mathrm{H} / \mathrm{R}+\mathrm{H}+\mathrm{A})$ group; and vi) the $\mathrm{H} / \mathrm{R}+5 \mu \mathrm{M} \mathrm{S} 1491+20 \mu \mathrm{M}$ HSYA (H/R + H + S1491) group. As previously described and following our preliminary experiments, the doses of each agent were selected (29-32). The cells were collected for the following experiments.

Infarct size measurement. As described previously, the risk area and infarct size was calculated using a double-staining method with TCC and Evans blue stains (33). In brief, following perfusion, the heart was perfused with $3 \%$ Evans blue to indicate the area at risk (AAR). Then, the tissues were incubated with $2 \%$ TTC solution in the dark at $37^{\circ} \mathrm{C}$ for $15 \mathrm{~min}$, and then were then stored in $4 \%$ paraformaldehyde overnight at $4^{\circ} \mathrm{C}$ to demarcate the infarct area. Areas with 
non-perfusion and blue staining were considered AAR, while areas with non-perfusion with TTC staining were labeled as the infarct area. The AAR and infarct area were calculated using Image-Pro Plus software V6.0 (Media Cybernetics, Inc., Rockville, MD, USA). The infarct size was measured as a percentage of the infarct area over the AAR.

Analysis of myocardial damage and oxidative stress markers. Following reperfusion, blood samples in rats and cells in the culture medium were harvested. The blood and cells were then centrifuged at $3,000 \mathrm{x} \mathrm{g}$ for $15 \mathrm{~min}$ at room temperature to obtain sera and cell supernatant. The levels of cTnI, IL-6 and $\mathrm{LDH}$ release were examined to estimate myocardial damage. The levels were measured using commercially available ELISA kits according to the manufacturer's protocol. The absorbance was read on a microplate reader (Thermo Fisher Scientific, Inc.). The serum and the cell culture supernatant were also used to estimate oxidative stress markers, including the content of MDA and the activity of SOD, which were detected with commercial assay kits using fluorescence spectrophotometry (F-7100; Hitachi, Ltd., Tokyo, Japan) following the manufacturer's protocols.

Detection of myocardial apoptosis. A terminal deoxynucleotidyl transferase-mediated dUTP nick-end labeling (TUNEL) assay and flow cytometry analysis were performed to detect the apoptosis levels in heart tissue and cardiomyocytes, respectively. The heart tissue was fixed in $4 \%$ paraformaldehyde for $10 \mathrm{~min}$ at room temperature and embedded in paraffin. The tissue was subsequently cut into $4-5 \mu \mathrm{m}$ sections, and the slides were incubated with TUNEL mixture reagent (Sigma-Aldrich; Merck KGaA) at $37^{\circ} \mathrm{C}$ for $1 \mathrm{~h}$. The cells were also incubated with DAPI $(1 \mu \mathrm{g} / \mathrm{ml})$ for $30 \mathrm{~min}$ at room temperature to stain the nuclei. ProLong ${ }^{\mathrm{TM}}$ Gold Antifade Mountant (cat. no. P36930; Thermo Fisher Scientific, Inc.) was used to prevent photo-bleaching. The apoptotic cells in $\geq 6$ fields were randomly selected. The percentage of apoptosis was calculated as the number of TUNEL-positive cells within the total populations of cardiomyocytes. The images and the ratios were obtained using an Olympus fluorescence microscope (BX51; Olympus Corporation, Tokyo, Japan) and ImageJ software (version 2.0.0; National Institutes of Health, Bethesda, MD, USA), respectively. An Annexin V-fluorescein isothiocyanate (FITC) and propidium iodide (PI) staining kit (BD Biosciences, San Jose, CA, USA) was used to assess cell apoptosis in vitro according to the manufacturer's protocols. The collected cells were stained with $5 \mu$ l Annexin V-FITC and $10 \mu \mathrm{l} \mathrm{PI}$ in the dark at room temperature for 10-15 min. Apoptosis was determined by a FACSCalibur flow cytometer (BD Biosciences) and analysis software BD CellQuest ${ }^{\mathrm{TM}}$ Pro Software version 1.2 (BD Biosciences).

Cell viability and caspase -3 activity. The cells $\left(5 \times 10^{3}\right.$ per well) were seeded in a 96-well plate and treated as described. Next, the cells were incubated with Cell Counting Kit-8 (CCK-8; Beyotime Institute of Biotechnology) reagent $(10 \mu \mathrm{l})$ at $37^{\circ} \mathrm{C}$ in an incubator for $3 \mathrm{~h}$. The absorbance was determined on a microplate reader (Bio-Rad Laboratories, Inc., Hercules, CA, USA) at $450 \mathrm{~nm}$. Caspase-3 activity (cat. no. 69-21755; MSK Laboratory, Wuhan, China) was determined in the cell lysate by conducting an ELISA assay. The treated cells were collected and plated in a 96-well plate. Cell lysates were subject to protein quantitation using a Bicinchoninic Acid Protein Assay kit (Bio-Rad Laboratories, Inc.) prior to detection. Each well was incubated with substrate solution. Then, the 96-well plate was maintained at $37^{\circ} \mathrm{C}$ for $30 \mathrm{~min}$. Finally, the reaction was terminated with a stop solution. A microplate reader was used to read the absorbance at a wavelength of $405 \mathrm{~nm}$.

Determination of mitochondrial membrane potential (MMP) and ROS generation. The alteration of MMP was measured by Rhodamine-123 (Rho-123) dye (Sigma-Aldrich; Merck $\mathrm{KGaA}$ ). Following reperfusion, the cells were incubated with $10 \mu \mathrm{g} / \mathrm{ml} \mathrm{Rho-123}$ working buffer at $37^{\circ} \mathrm{C}$ for $30 \mathrm{~min}$. The cells were then washed, and analyzed using a flow cytometer and BD Accuri ${ }^{\mathrm{TM}}$ C6 software (BD Biosciences). Subsequently, 2,7-dichlorofluorescein diacetate (DCFH-DA) dye (Sigma-Aldrich; Merck KGaA) was used to detect the ROS level. Briefly, prior to fixation with $4 \%$ paraformaldehyde at room temperature for $15 \mathrm{~min}$, the cells were stained with $20 \mu \mathrm{M} \mathrm{DCFH}-\mathrm{DA}$ at $37^{\circ} \mathrm{C}$ for $30 \mathrm{~min}$. ROS generation was then determined at wavelength $488 \mathrm{~nm}$ using a fluorescence plate reader (BioTek Instruments, Inc., Winooski, VT, USA).

Reverse transcription quantitative polymerase chain reaction $(R T-q P C R)$. Cells were pretreated as described. The total RNA was isolated with TRIzol ${ }^{\circledR}$ reagent (Thermo Fisher Scientific, Inc.). DNaseI was used to digest the genomic DNA. Reverse transcription was completed with random hexamer primers using a Moloney-Murine Leukemia Virus Enzyme (Promega Corporation, Madison, WI, USA). RT-qPCR was performed on ABI Prism 7500 using the SYBR Premix Ex Taq II (Takara Bio, Inc., Otsu, Japan). The qPCR conditions were set as follows: $10 \mathrm{~min}$ pretreatment at $95^{\circ} \mathrm{C}, 96^{\circ} \mathrm{C}$ for $15 \mathrm{sec}, 63^{\circ} \mathrm{C}$ for $45 \mathrm{sec}$ ( 35 cycles) and a final extension at $75^{\circ} \mathrm{C}$ for $10 \mathrm{~min}$. The specific PCR primers used were as follows: Fas forward, 5'-CCAGCCACAAAGAGAGGAGA-3'; Fas reverse, 5'-AACGGTTCCTCTCAACACCT-3'; FasL forward, 5'-TGCTGTGTGACAATGCAGAG-3', FasL reverse, 5'-GAG CCTCCTTTCTCACCCTT-3'; Bcl-2 forward, 5'-CCTGGC ATCTTCTCCTTCCA-3'; Bcl-2 reverse, 5'-GGACATCTC TGCAAAGTCGC-3'; Bax forward, 5'-TGGCCTCCTTTC CTACTTCG-3'; Bax reverse, 5'-AAAATGCCTTTCCCCGT TCC-3'; $\beta$-actin forward, 5'-TCTATGAGGGTTACGCGCTC-3'; $\beta$-actin reverse, 5'-GCTGTGGTGGTGAAGCTGTA-3'. The $2^{-\Delta \Delta \mathrm{Cq}}$ method was used to quantify gene expression levels (34).

Western blot analysis. The harvested cells were treated with radioimmunoprecipitation assay lysis buffer (Beyotime Institute of Biotechnology). A Bicinchoninic Acid Protein Assay kit (Bio-Rad Laboratories, Inc.) was used to assess protein concentration. Following denaturation in boiling water for $5 \mathrm{~min}$, the proteins $(20 \mu \mathrm{g} /$ lane) were separated by electrophoresis on $10 \%$ SDS-PAGE gels. Then, the cells were transferred onto a polyvinylidene fluoride membrane. Subsequently, 5\% skimmed milk was incubated with the membrane to block the non-specific antigens at room temperature for $2 \mathrm{~h}$. The primary antibodies were then added, to interact with the target proteins, at $4^{\circ} \mathrm{C}$ overnight. Next, the membrane was incubated with HRP-coupled secondary antibodies at $4^{\circ} \mathrm{C}$ for $1 \mathrm{~h}$. Blots were visualized 
A

Control
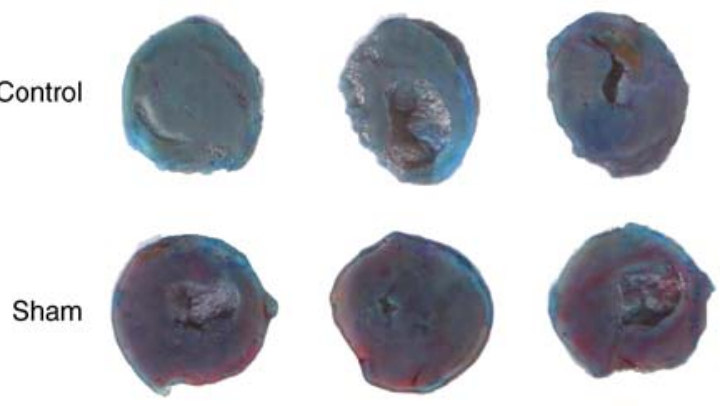

I/R
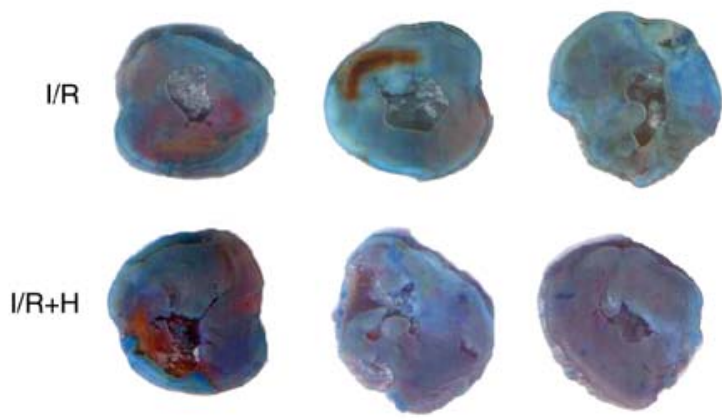

$1 \mathrm{~cm}$

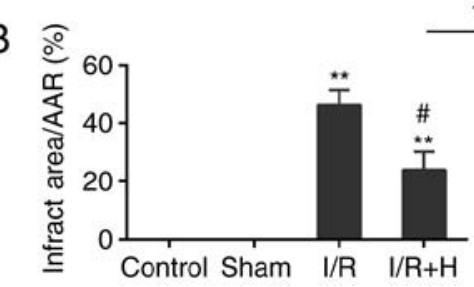

Figure 1. Myocardial infarct size in I/R rats is decreased by HSYA. (A) The effect of HSYA was examined by double-staining technique of 2,3,5-triphenyltetrazolium chloride and Evans blue. (B) The percentage of the AAR (IS/AAR\%) was indicated the infarct size. ${ }^{* *} \mathrm{P}<0.01$ vs. control group; ${ }^{*} \mathrm{P}<0.05$ vs. I/R group. AAR, area at risk; I/R, ischemia/reperfusion; H/HSYA Hydroxysafflor Yellow A.

using enhanced chemiluminescence reagent (Thermo Fisher Scientific, Inc.) An enhanced chemiluminescence system (GE Healthcare, Chicago, IL, USA) was adopted to detect the bands. The protein gray intensity was calculated using Quantity one software (4.6.2; Bio-Rad Laboratories, Inc.).

Statistical analysis. GraphPad Prism Software 6 (GraphPad Software, Inc., La Jolla, CA, USA) was used for statistical analysis. Statistical analysis was conducted by one-way analysis of variance with Dunnett's post-test comparison. Data are presented as the mean \pm standard deviation from three independent experiments. $\mathrm{P}<0.05$ was considered to indicate a statistically significant difference.

\section{Results}

HSYA ameliorates myocardial I/R injury and oxidant stress in vivo. Infarct size and the release of cTnI and IL-6 were measured to estimate the effect of HSYA on I/R injury. In contrast to the Sham group, animals in the I/R group exhibited a significant increase in myocardial infarct size. The infarct size was markedly decreased in the HSYA group in comparison with that in the I/R group (Fig. 1A and B). In addition, animals in the I/R group exhibited a marked increase in serum cTnI and IL-6 levels, compared with those rats in the Sham group.

Notably, the treatment with HSYA was associated with a minor increase in cTnI and IL-6 levels compared with the I/R group (Fig. 2A and B). Furthermore, as a marker of tissue damage, the increased activity of LDH induced by I/R injury was decreased by the treatment with HSYA (Fig. 2C). In addition, the animals in the I/R group exhibited a noticeable increase in the content of MDA and a significant decrease in SOD activity compared with the Sham group. The administration of HSYA in animals with I/R injury exhibited cardio-protective effects by alleviating oxidative stress (Fig. 2D and E).

HSYA decreases myocardial apoptosis in vivo. To estimate the effect of HSYA on apoptosis, the TUNEL-positive cardiomyocytes and the expression levels of apoptosis-associated proteins were determined in vivo. The percentage of TUNEL-positive cells was elevated in the I/R group in comparison with the Sham group. Compared with the I/R group, HSYA significantly decreased the number of TUNEL-positive cardiomyocytes (Fig. 3A and B). In addition, the expression levels of pro-apoptotic proteins, including cleaved caspase-3, Fas, FasL and Bax, were markedly induced by I/R. However, the administration of HSYA decreased their expression levels. Concomitantly, the level of anti-apoptotic protein Bcl-2 was decreased in the $\mathrm{I} / \mathrm{R}$ group, but it was recovered in the $\mathrm{I} / \mathrm{R}+\mathrm{HSYA}$ group (Fig. 3C and D).

HSYA suppresses myocardial p-JAK2 and p-STAT1 protein expression levels. The expression levels of p-JAK2 and p-STAT1 were markedly increased in animals with I/R injury. No significant difference in the expression levels of total JAK2 and STAT1 between Sham and I/R group was identified. The administration of HSYA decreased the levels of p-JAK2 and p-STAT1 (Fig. 4A-D).

Inhibition of JAK2/STAT1 signaling HSYA prevents $H 9 c 2$ cardiomyocytes against I/R injury. CCK-8 data indicated that compared with the control group, the cell viability was significantly inhibited following I/R injury, while pretreatment with HSYA or inhibitors of JAK2/STAT1 (AG490 or S1491) significantly rescued the cell viability (Fig. 5A). In addition, the release of cTnI and IL-6 was decreased in the H/R group compared with the control group, while such a release was decreased by the pretreatment of HSYA, AG490 or S1491 (Fig. 5B and C). The protective effect appeared more apparent in the $\mathrm{HR}+\mathrm{A}+\mathrm{H}$ or $\mathrm{H} / \mathrm{R}+\mathrm{S}+\mathrm{H}$ group compared with the $\mathrm{H} / \mathrm{R}+\mathrm{H}$ group.

Inhibition of JAK2/STAT1 signaling strengthens the antioxidant effects of HSYA in H9c2. Data from the assays for MDA content and SOD activity demonstrated that I/R injury accelerated the rate of oxidative stress compared with the control. The MDA content and SOD activity were markedly decreased and recovered, respectively, in the group pretreated with HSYA. Although no significant difference was identified in the MDA content and SOD activity in $\mathrm{H} / \mathrm{R}+\mathrm{S}+\mathrm{H}$ group when compared with those in the $\mathrm{H} / \mathrm{R}+\mathrm{H}$ group, the protective effects of HSYA were more significantly enhanced by the combined use with AG490 or S1491 (Fig. 6A and B). Loss of MMP may be associated with the disruption of redox status, which then leads to ROS-triggered oxidative stress. The results 

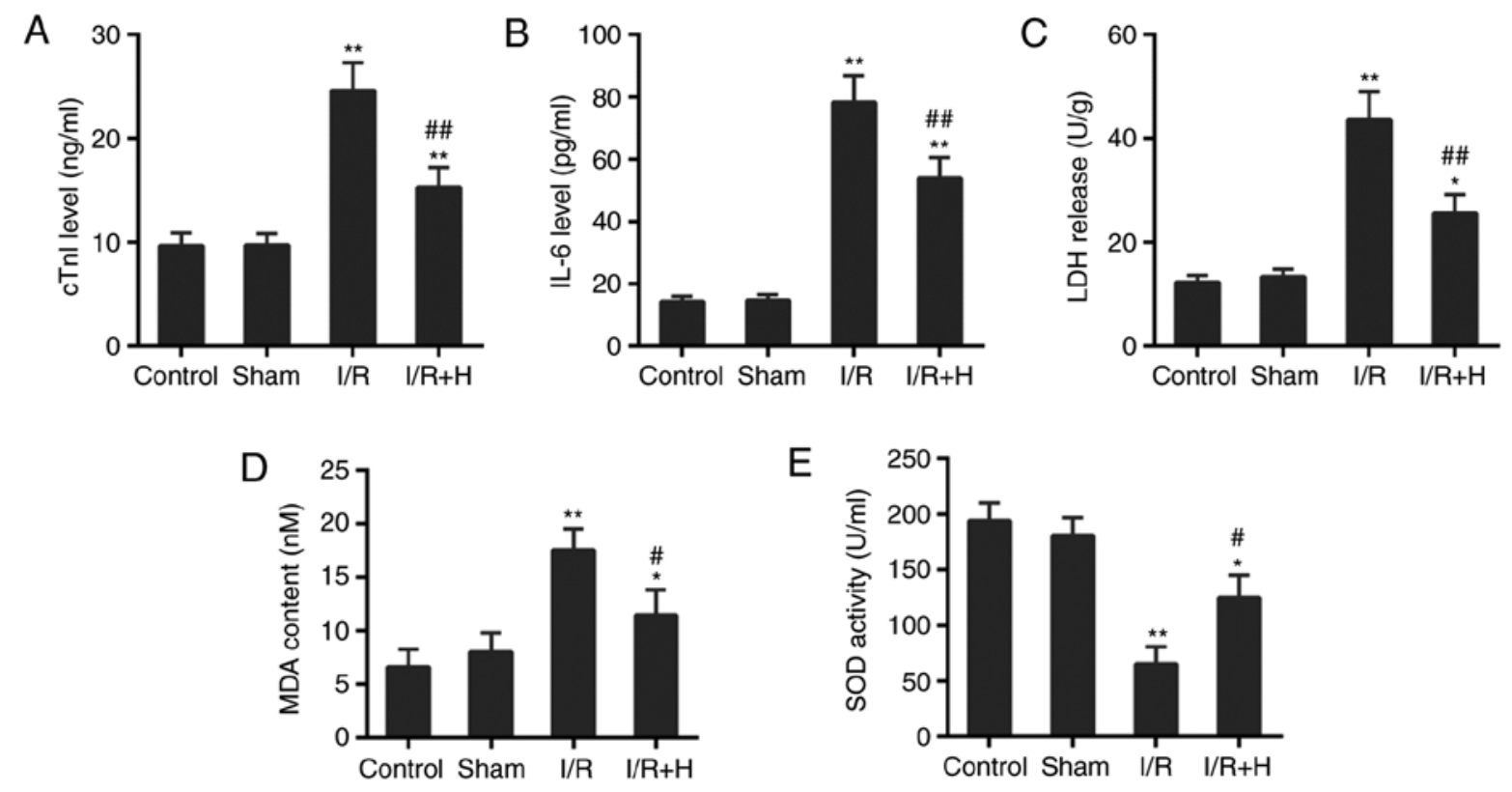

Figure 2. Effect of HSYA on indicators for myocardial injury and oxidative stress. The serum levels of (A) cTnI, (B) IL-6, (C) LDH, (D) MDA and (E) SOD in rats. $\mathrm{P}<0.05$ and ${ }^{* * *} \mathrm{P}<0.01$ vs. control group; ${ }^{\#} \mathrm{P}<0.05$ and ${ }^{\# \#} \mathrm{P}<0.01$ vs. I/R group. I/R, ischemia/reperfusion; H/HSYA, Hydroxysafflor Yellow A; cTnI, cardiac troponin; IL-6, interleukin 6; LDH, lactate dehydrogenase; MDA, malondialdehyde; SOD, superoxide dismutase.

A
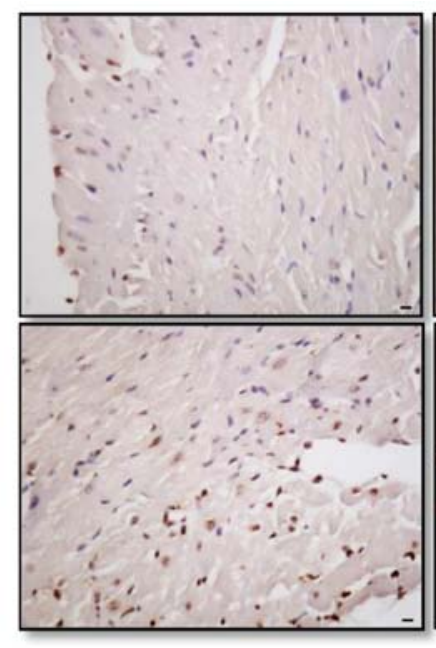

C

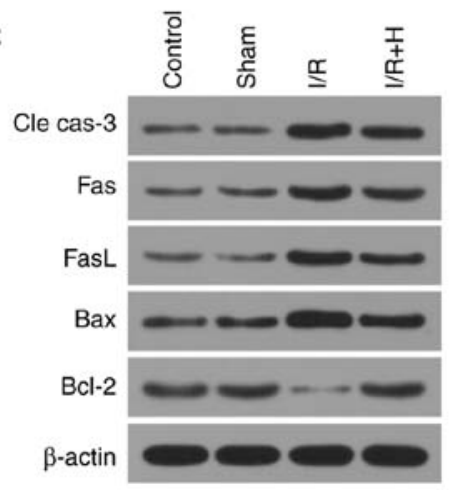

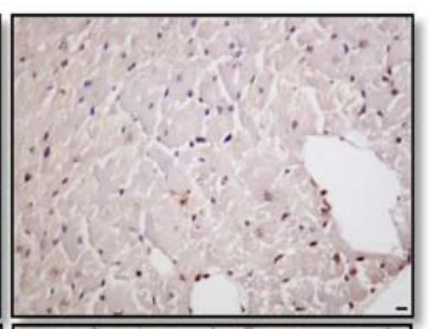

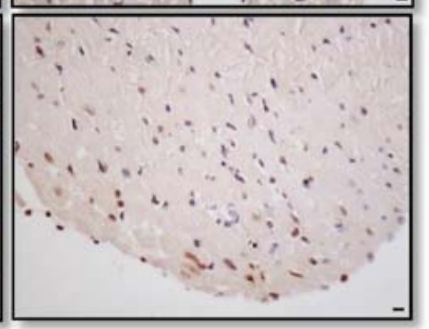

B

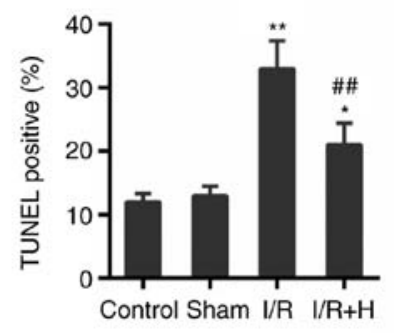

Figure 3. Effect of HSYA on myocardial apoptosis. (A) Representative TUNEL staining images in each group. Scale bar=100 $\mu$ M. (B) Apoptosis was determined by the number of TUNEL-positive cells. (C) Western blot analysis for the protein expression levels of cle cas-3, Fas, FasL, Bax and Bcl-2. (D) Densitometric analysis of the western blot analysis data. ${ }^{*} \mathrm{P}<0.05$ and ${ }^{* *} \mathrm{P}<0.01$ vs. control group; ${ }^{\#} \mathrm{P}<0.05$ and ${ }^{\# \#} \mathrm{P}<0.01$ vs. I/R group. I/R, ischemia/reperfusion; H/HSYA, Hydroxysafflor Yellow A; TUNEL, terminal deoxynucleotidyl transferase-mediated dUTP nick-end labeling; cle cas-3, cleaved caspase-3; Fas, tumor necrosis factor receptor superfamily member 6; FasL, Fas ligand; Bcl-2, B-cell lymphoma 2; Bax, Bcl-2-associated X protein. 

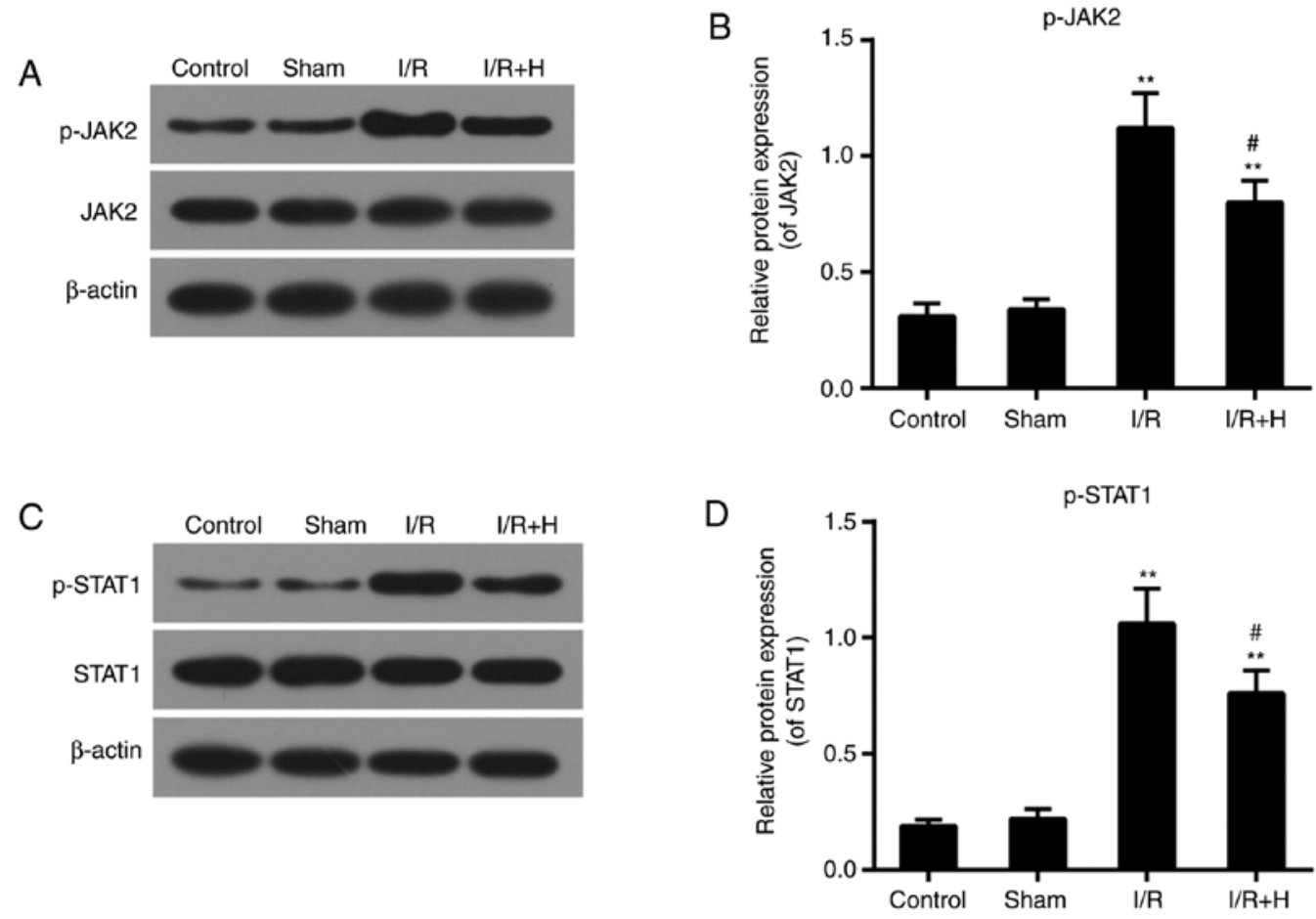

Figure 4. Effect of HSYA on the JAK2/STAT1 pathway. (A) Western blot analysis of the protein expression levels of JAK2 and p-JAK2. (B) Quantification of the western blot analysis data. (C) Western blot for the protein expression levels of STAT1 and p-STAT1. (D) Quantification of the western blot analysis data. ${ }^{* *} \mathrm{P}<0.01$ vs. control group; ${ }^{~} \mathrm{P}<0.05$ vs. I/R group. I/R, ischemia/reperfusion; H/HSYA, Hydroxysafflor Yellow A; JAK2, janus kinase 2; STAT1, signal transducer and activator of transcription 1; p, phosphorylated.
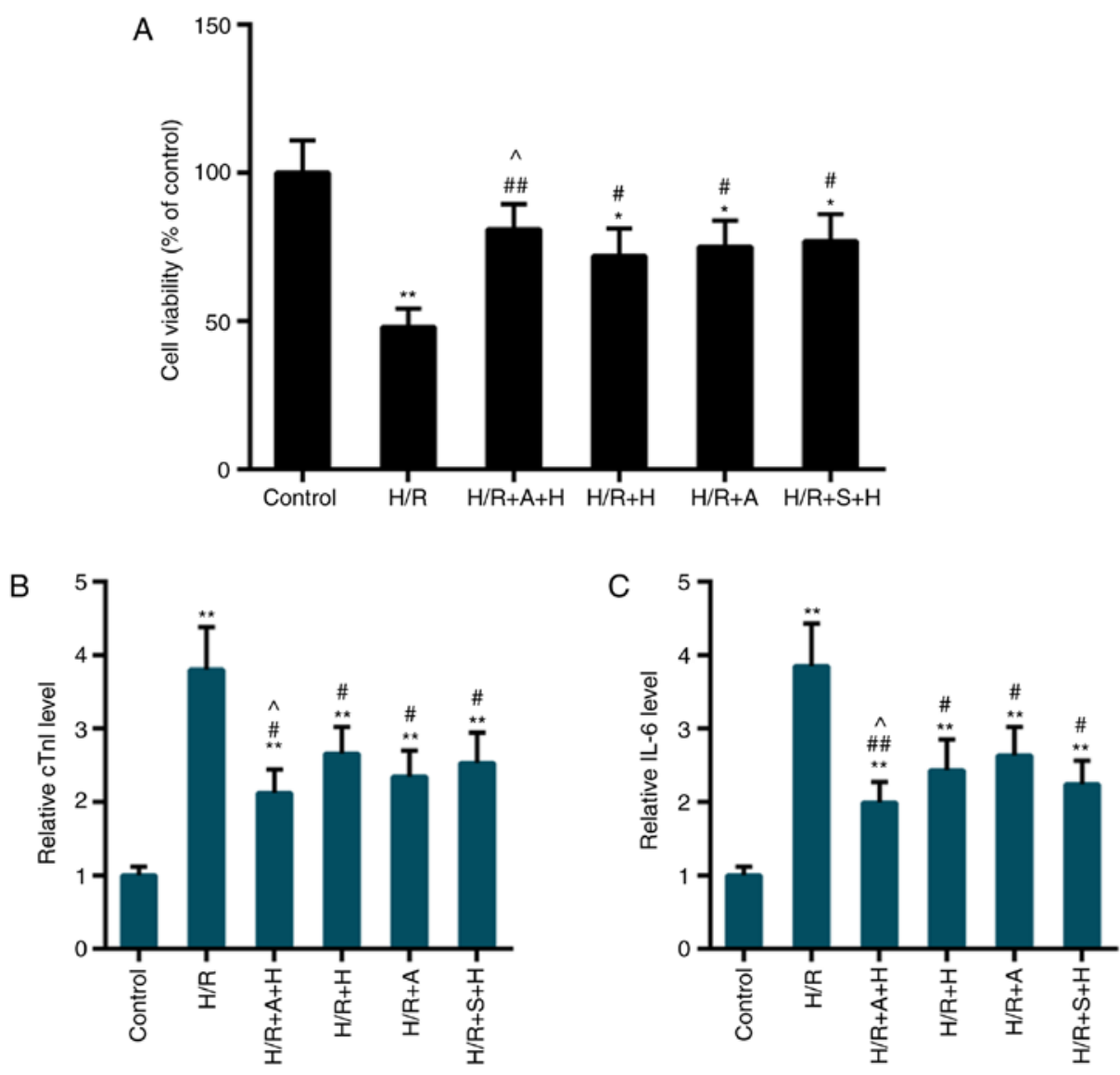

Figure 5. Effect of inhibition of janus kinase 2/signal transducer and activator of transcription 1 on myocardial injury in vitro. (A) The cell viability was detected by Cell Counting Kit- 8 method. The levels of (B) cTnI and (C) IL- 6 were estimated by ELISA. ${ }^{*} \mathrm{P}<0.05$ and ${ }^{* *} \mathrm{P}<0.01$ vs. control group; ${ }^{*} \mathrm{P}<0.05$ and ${ }^{\# \#} \mathrm{P}<0.01$ vs. $\mathrm{H} / \mathrm{R}$ group; ${ }^{\wedge} \mathrm{P}<0.05$ vs. H/R+H group. H/R, hypoxia/reoxygenation; H, Hydroxysafflor Yellow A; A, AG490; S, S1491; cTnI, cardiac troponin; IL-6, interleukin 6 . 
A

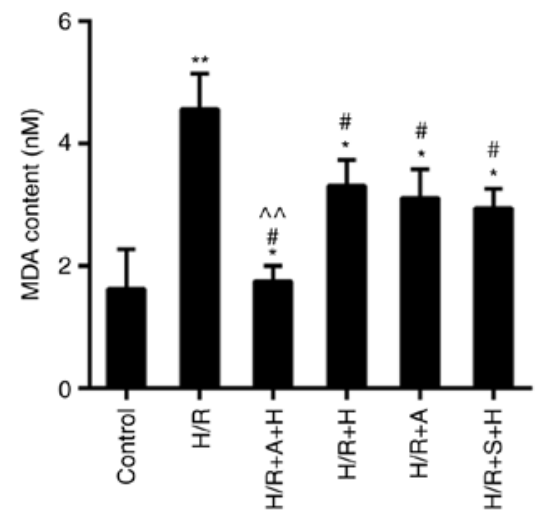

B

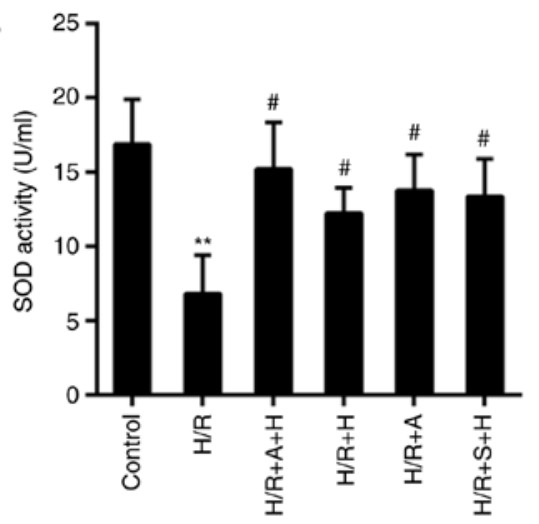

C
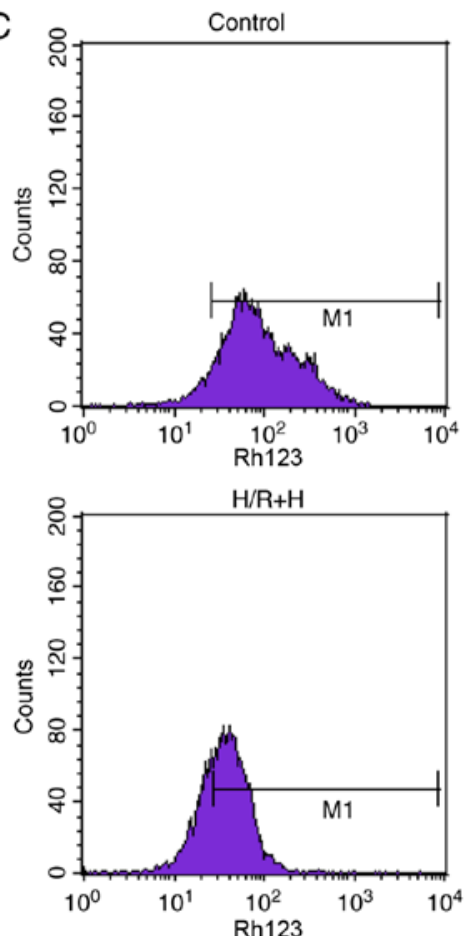
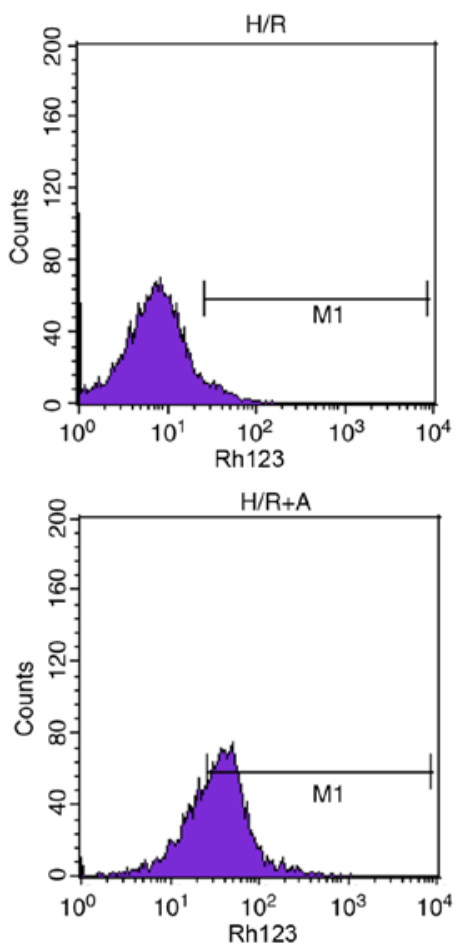
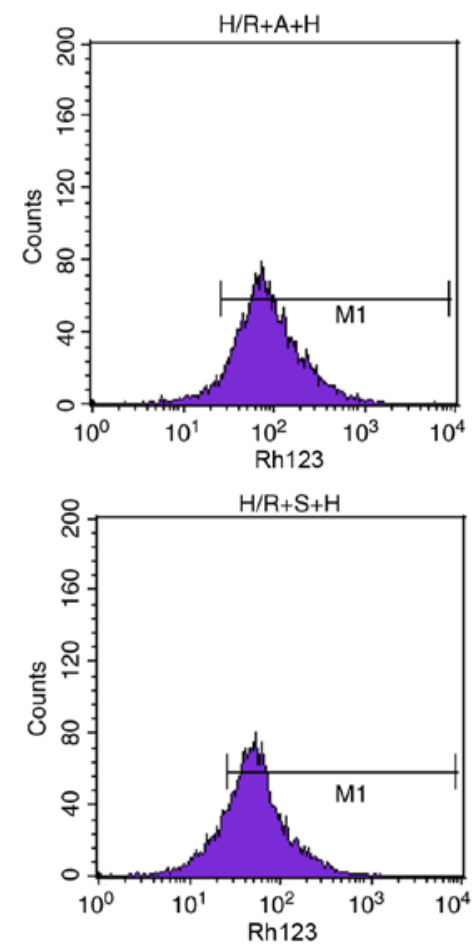
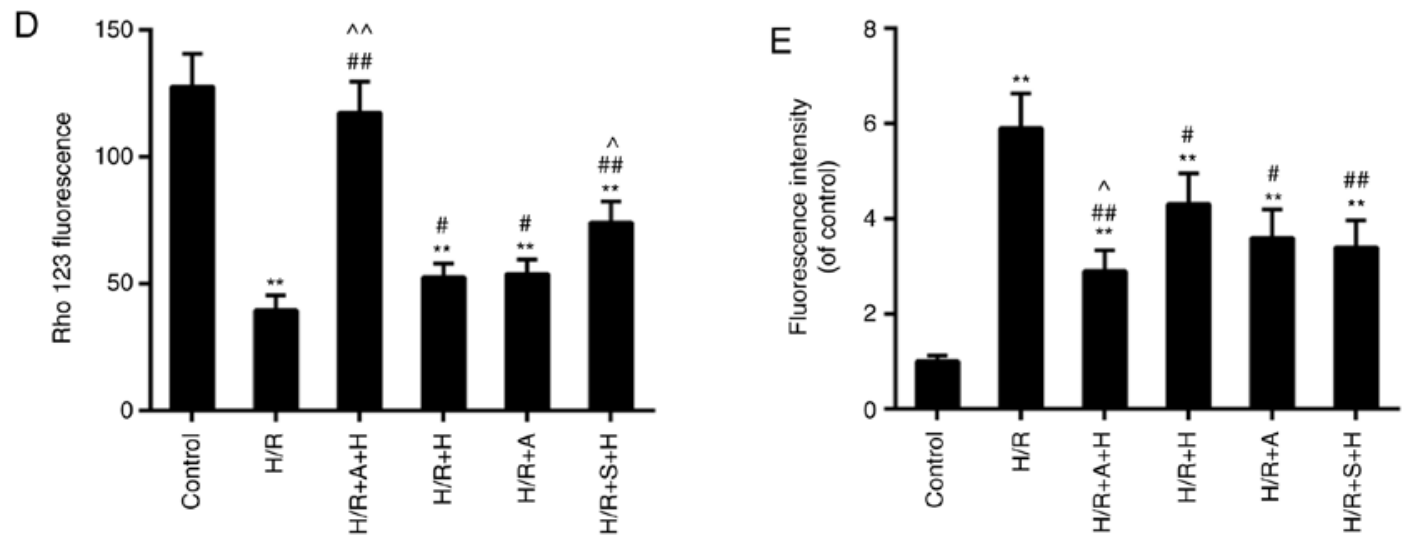

Figure 6. Effect of inhibition of janus kinase 2/signal transducer and activator of transcription 1 on oxidative stress in vitro. The release of (A) MDA and (B) SOD. (C) The alteration of mitochondrial membrane potential was examined by flow cytometry analysis. (D) Quantification of Rho 123 cytometry data. (E) The reactive oxygen species production in $\mathrm{H} 9 \mathrm{c} 2$ cells was detected by 2,7 -dichlorofluorescein diacetate fluorescence signals. ${ }^{*} \mathrm{P}<0.05$ and ${ }^{* *} \mathrm{P}<0.01$ vs. control group; ${ }^{\#} \mathrm{P}<0.05$ and ${ }^{\# \#} \mathrm{P}<0.01$ vs. $\mathrm{H} / \mathrm{R}$ group; ${ }^{\wedge} \mathrm{P}<0.05$ and ${ }^{\wedge} \mathrm{P}<0.01$ vs. $\mathrm{H} / \mathrm{R}+\mathrm{H}$ group. $\mathrm{H} / \mathrm{R}$, hypoxia/reoxygenation; H, Hydroxysafflor Yellow A; A, AG490; S, S1491; MDA, malondialdehyde; SOD, superoxide dismutase; Rho 123, rhodamine 123.

indicated that the loss of MMP was all partially alleviated in $\mathrm{H} / \mathrm{R}+\mathrm{A}+\mathrm{H}, \mathrm{H} / \mathrm{R}+\mathrm{H}, \mathrm{H} / \mathrm{R}+\mathrm{A}$ and $\mathrm{H} / \mathrm{R}+\mathrm{S}+\mathrm{H}$ groups, compared with the control group. Consistently, the ROS contents in these groups were decreased compared with those in the control group. The ROS level was significantly decreased in the $\mathrm{H} / \mathrm{R}+\mathrm{A}+\mathrm{H}$ and $\mathrm{H} / \mathrm{R}+\mathrm{S}+\mathrm{H}$ groups, compared with 

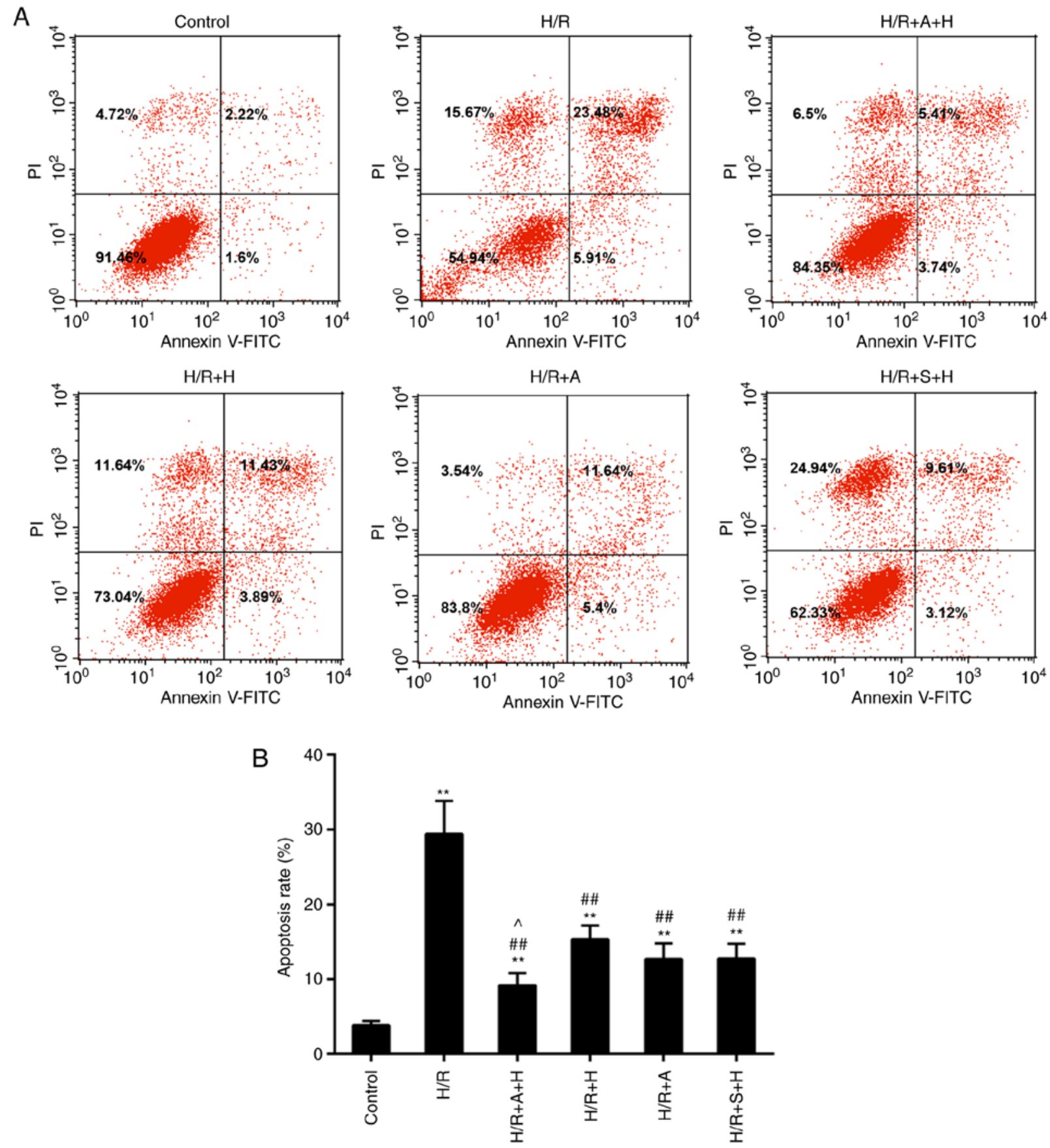

Figure 7. Effect of inhibition of janus kinase 2/signal transducer and activator of transcription 1 on apoptosis in vitro. (A) The apoptosis of $\mathrm{H} 9 \mathrm{c} 2$ was assessed by flow cytometry analysis. (B) Quantification of flow cytometry data. ${ }^{* *} \mathrm{P}<0.01$ vs. control group; ${ }^{\# \#} \mathrm{P}<0.01$ vs. $\mathrm{H} / \mathrm{R}$ group; ${ }^{\wedge} \mathrm{P}<0.05$ vs. $\mathrm{H} / \mathrm{R}+\mathrm{H}$ group. $\mathrm{H} / \mathrm{R}$, hypoxia/reoxygenation; H, Hydroxysafflor Yellow A; A, AG490; S, S1491; PI, propidium iodide; FITC, fluorescein isothiocyanate.

the $\mathrm{H} / \mathrm{R}+\mathrm{H}$ group. Although no significant difference was identified in the $\mathrm{H} / \mathrm{R}+\mathrm{S}+\mathrm{H}$ group in comparison with that in the $\mathrm{H} / \mathrm{R}+\mathrm{H}$ group, the MMP level was also decreased in the $\mathrm{H} / \mathrm{R}+\mathrm{A}+\mathrm{H}$ and $\mathrm{H} / \mathrm{R}+\mathrm{S}+\mathrm{H}$ groups, compared with the $\mathrm{H} / \mathrm{R}+\mathrm{H}$ group (Fig. $6 \mathrm{C}-\mathrm{E})$.

Inhibition of JAK2/STAT1 signaling enhances the anti-apoptotic effect of HSYA in H9c2. To confirm the effect of HSYA on apoptosis, the levels of apoptosis were detected by flow cytometry. The results revealed that the apoptosis rate was accelerated in the $\mathrm{H} / \mathrm{R}$ group, while decreased cell apoptosis was identified in the $\mathrm{H} / \mathrm{R}+\mathrm{A}+\mathrm{H}, \mathrm{H} / \mathrm{R}+\mathrm{H}, \mathrm{HR}+\mathrm{A}$ and $\mathrm{H} / \mathrm{R}+\mathrm{S}+\mathrm{H}$ groups compared with the H/R group (Fig. 7A and B). Furthermore, the caspase- 3 activity was decreased in the $H / R+A+H$, $\mathrm{H} / \mathrm{R}+\mathrm{H}, \mathrm{HR}+\mathrm{A}$ and $\mathrm{H} / \mathrm{R}+\mathrm{S}+\mathrm{H}$ groups compared with the H/R group (Fig. 8A). Additionally, the expression levels of apoptosis-associated factors were evaluated. The data from the in vitro analyses were compatible with those from the in vivo experiments, which demonstrated that the expression levels of pro-apoptotic proteins (cleaved caspase-3, Fas, FasL and Bax) were decreased and anti-apoptotic protein $\mathrm{Bcl}-2$ was increased in the $\mathrm{H} / \mathrm{R}+\mathrm{A}+\mathrm{H}, \mathrm{H} / \mathrm{R}+\mathrm{H}, \mathrm{H} / \mathrm{R}+\mathrm{A}$ and $\mathrm{H} / \mathrm{R}+\mathrm{S}+\mathrm{H}$ groups compared with that in the H/R group (Fig. $8 \mathrm{~B}$ and $\mathrm{C}$ ). Similar results were obtained with regards to the mRNA expression 

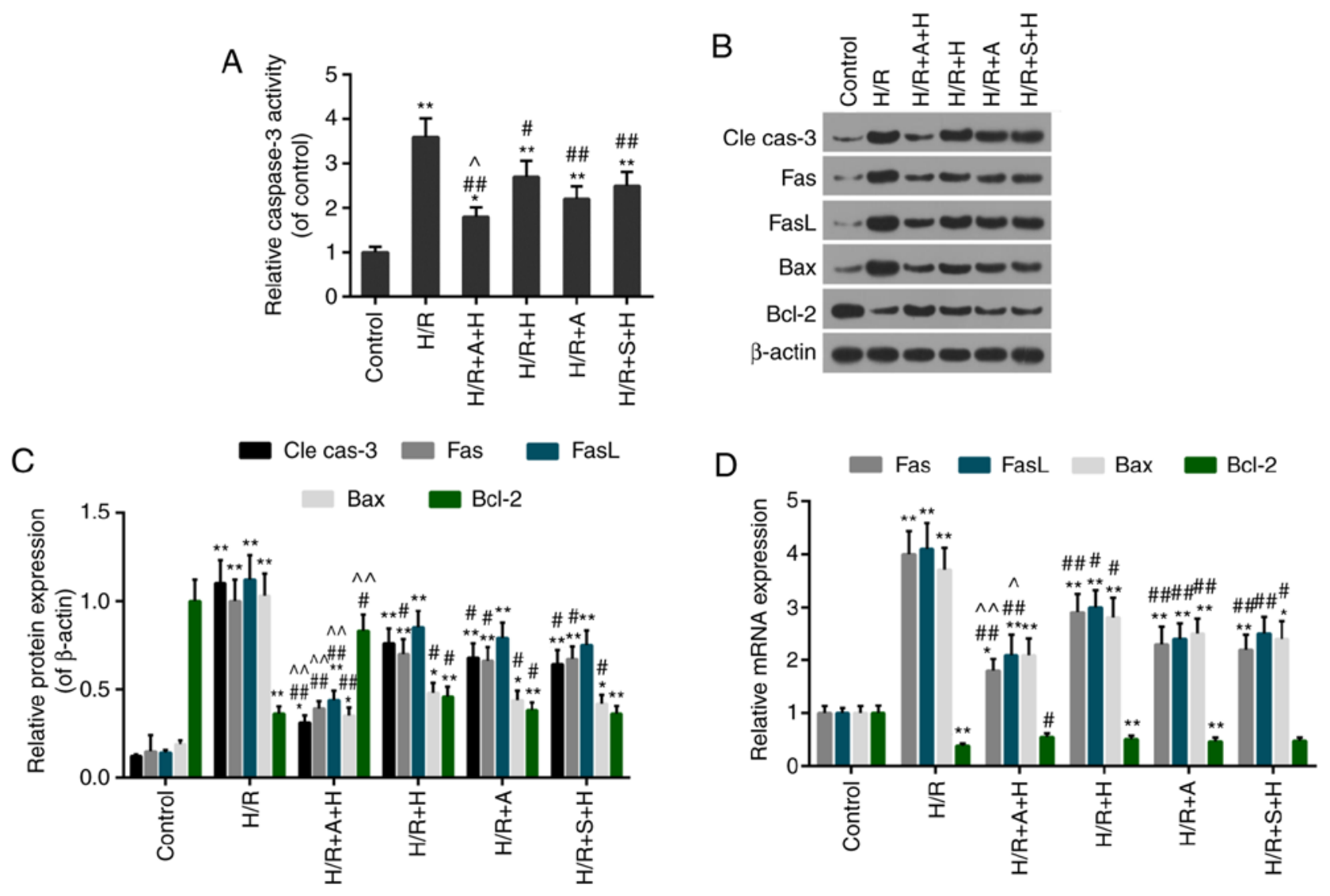

Figure 8. Effect of inhibition of janus kinase 2/signal transducer and activator of transcription 1 on the activity of apoptosis-associated proteins. (A) Caspase-3 activity was estimated using an ELISA kit. (B) Western blot analysis gel examining the protein levels of cle cas-3, Fas, FasL, Bax and Bcl-2. (C) Quantification of the western blot analysis data. (D) Reverse transcription quantitative polymerase chain reaction assay of the expression levels of Fas, FasL, Bax and Bcl-2. ${ }^{*} \mathrm{P}<0.05$ and ${ }^{* *} \mathrm{P}<0.01$ vs. control group; ${ }^{\#} \mathrm{P}<0.05$ and ${ }^{\# \#} \mathrm{P}<0.01$ vs. HR group; ${ }^{\wedge} \mathrm{P}<0.05$ and ${ }^{\wedge} \mathrm{P}<0.01 \mathrm{vs}$. H/R+H group. cle cas-3, cleaved caspase-3; H/R, hypoxia/reoxygenation; H, Hydroxysafflor Yellow A; A, AG490; S, S1491; Fas, tumor necrosis factor receptor superfamily member 6; FasL, Fas ligand; Bcl-2, B-cell lymphoma 2; Bax, Bcl-2-associated X protein.

levels of these apoptosis-associated factors (Fig. 8D). Therefore, the treatment with AG490/S1491 enhanced the protective effect of HSYA.

\section{Discussion}

I/R injuries are one of the primary causes of MI (3). HSYA, the primary active component of Carthamus tinctorius L., has been suggested to be able to protect against multiple types of cell injuries $(35,36)$. In the present study, the protective role of HSYA was demonstrated, following I/R injury in an in vivo animal model and in vitro experiments using cultured H9c2 cells. The signaling pathway by which HSYA delivers its protective effect was also explored.

Infarct size is considered an important index in determining the severity of I/R injury in heart muscle (37). Therefore, the present study first determined the effects of HSYA on infarct size. With the administration of HSYA, the infarct size was decreased compared with the Sham group. In addition, the levels of cTnI and IL-6, sensitive markers of cardiac injury (38), were decreased in the I/R+HSYA treatment group compared with the I/R group. Furthermore, the release of $\mathrm{LDH}$, which may reflect the degree of $\mathrm{I} / \mathrm{R}$ damage, was also decreased following pretreatment with HSYA, indicating that HSYA is capable of decreasing myocardial injury caused by I/R in vivo.
The mechanism by which HSYA realized its protective effects was then investigated. The increased production of free radicals and the decreased activities of antioxidant enzymes are closely associated with myocardial I/R injury (39). The disrupted redox status will trigger oxidative stress (OS). SOD and MDA are common indicators of OS. The former is responsible for decomposing $\mathrm{O}_{2}$ and $\mathrm{H}_{2} \mathrm{O}_{2}$, while the latter is the product of lipid peroxidation $(40,41)$. The results from the present study revealed that these indicators of oxidative stress were alleviated by HSYA, indicating an antioxidant scavenger capacity of HSYA.

Apoptosis is involved in the destruction of cardiomyocytes following I/R injury $(29,42)$. It has been recognized that Bcl-2 and Bax are essential proteins in apoptosis (29). The $\mathrm{Bax} / \mathrm{Bcl}-2$ ratio regulates the cell sensitivity death signals by modulating the function of the mitochondria. Caspase- 3 is a key gene of the apoptotic pathways and may be processed into cleaved caspase-3 (43). Additionally, the Fas/FasL death receptor-mediated pathway may be activated in I/R injury (11-14). In the present study, HSYA decreased cleaved caspase-3, Fas and FasL levels, increased the Bcl-2/Bax ratio and mitigated TUNEL-positive staining in comparison with the I/R group. Taken together, the results demonstrated an anti-apoptotic effect of HSYA following $\mathrm{I} / \mathrm{R}$ injury. 
Subsequently, the present study aimed to explore the molecular signaling methods by which HSYA exerted its effective role. The JAK/STAT pathway is important in signal transduction and is involved in mediating cell growth, proliferation and differentiation, and apoptosis. JAK and STAT family members are located in myocardial cells and are closely associated with myocardial I/R injury (44). STAT-1, a member of the STATs, is able to regulate intracellular signaling. It has been demonstrated that STAT1 was activated following myocardial ischemia (45). Therefore, the present study focused particularly on the potential role of JAK2/STAT1 in HSYA-mediated anti-MI activity. The activity of the JAK2/STAT1 pathway in I/R injury was determined. HSYA treatment was observed to prevent the phosphorylation of JAK2, and it also decreased the phosphorylation of downstream STAT1. Altogether, these results suggested that the protective effect of HSYA was, at least, partially dependent on suppressing the JAK2/STAT1 pathway.

JAK/STAT pathways are important signaling pathways in response to stress, including ischemia, hypoxia and oxidative stress $(46,47)$. JAK2 may be activated by ROS and hypoxic conditions (48). STAT-1 is phosphorylated by JAK2, and it serves a critical role in cerebral I/R injury in vivo (49). To additionally confirm the potential role of the JAK2/STAT1 signaling pathway in the HSYA-mediated protective effect, in vitro experiments using $\mathrm{H} 9 \mathrm{c} 2$ cells were performed in the present study. The data indicated that the inhibition of JAK2 (by AG490) or STAT1 (by S1491) enhanced the protective role of HSYA by improving cell viability and decreasing the release of cTnI, IL-6 and LDH. In addition, the HSYA-derived antioxidant capacity was strengthened by the inhibition of JAK2 or STAT1. The imbalance between the generation and removal of free radicals will lead to ROS accumulation. Mitochondria, the primary sites for ROS production, are associated with the loss of MMP (50). Therefore, the MMP and ROS content in $\mathrm{H} 9 \mathrm{c} 2$ was measured, and it was identified that the loss of MMP and the ROS generation were additionally deteriorated by H/R injury, while the HSYA treatment reversed this phenomenon. Notably, AG490 or S1491 increased the protective abilities of HSYA. Furthermore, the caspase-3 activity and the expression levels of cleaved caspase-3, Fas, FasL and Bax were additionally decreased in cells treated with AG490 or S1491, compared with the HSYA treatment alone. By contrast, the expression levels of Bcl-2 were increased in the groups treated with AG490 or S1491 compared with that in the $\mathrm{H} / \mathrm{R}+\mathrm{H}$ group. Additionally, examination of the apoptosis rate demonstrated that AG490 or S1491 treatment markedly enhanced the anti-apoptotic effect of HSYA, suggesting that HSYA and JAK2/STAT1 signaling have similar mechanisms of action. Previous studies suggested that the JAK/STAT signaling pathway may regulate apoptotic signals by decreasing caspase 3 protein expression and increasing anti-apoptotic Bcl-2 levels $(51,52)$. It has also been identified that JAK2 and STAT1 were activated following reperfusion, and that AG-490 may abrogate the activation of JAK2 $(47,53)$. The results from the present study were in concordance with these conclusions. The combined effects of HSYA and AG490/S1491 in an in vivo $\mathrm{I} / \mathrm{R}$ rat model would provide additional supporting evidence for the involvement of the JAK/STAT pathway in I/R injury. Altogether, the results from the present study indicated that
JAK2/STAT1 may serve as therapeutic targets to mitigate I/R injury. However, a previous study revealed that activating the JAK2/STAT3 signaling pathway may decrease HR-induced mitochondrial oxidative damage (54). We hypothesized that the discrepancy may be caused by distinct cell context and study model. In addition, previous studies have demonstrated that JAK2 may activate STAT3, STAT5a, STAT5b and STAT6 (55-57), therefore the role of these proteins in mediating the protective effects of HSYA in I/R injury should be additionally investigated in future studies.

Certain previous studies have documented the role of HSYA in myocardial IR injury (58-60). These studies suggested that HSYA exerted its cardio-protective effect by decreasing inflammation or oxidative stress via toll-like receptor 4 signaling or the extracellular signal-regulated kinase/glycogen synthase kinase $3 \beta$ pathway. This was a limitation of the present study. However, the mechanism of the protective effect of HSYA demonstrated in the present study was different from that in these previous studies. We hypothesized that these results together confirm the protective effects of HSAY on myocardial I/R injury and potentially provide insight for future clinical studies into HSYA.

In conclusion, HSYA attenuated myocardial injury in vitro and in vivo by inhibiting oxidative stress and apoptosis, in which the JAK2/STAT1 signaling pathway was partly involved. The results from the present study offered insight into the effects and the mechanisms of HSYA. It may provide effective therapeutic strategies to combat MI.

\section{Acknowledgements}

Not applicable.

\section{Funding}

The present study was supported by The Natural Science Foundation of Zhejiang Province, China (grant no. LY17H020001).

\section{Availability of data and materials}

The analyzed data sets generated during the study are available from the corresponding author on reasonable request.

\section{Authors' contributions}

DZ and TD made substantial contributions to the conception and design of the study. BL, YJ and SL contributed to data acquisition, and data analysis and interpretation. DZ and TD drafted the article and critically revised it for important intellectual content. All authors agreed to be accountable for all aspects of the work in ensuring that questions related to the accuracy or integrity of the work are appropriately investigate and resolved. All authors read and approved the final manuscript.

\section{Ethics approval and consent to participate}

The present study was performed according to the Guide for the Care and Use of Laboratory Animals, 8th Edition and 
approved by Animal Subjects Committee of the Affiliated Hospital of Hangzhou Normal University (Hangzhou, China).

\section{Patient consent for publication}

Not applicable.

\section{Competing interests}

The authors declare that they have no competing interests.

\section{References}

1. Bainey KR and Armstrong PW: Clinical perspectives on reperfusion injury in acute myocardial infarction. Am Heart J 167: 637-645, 2014.

2. Maxwell SR and Lip GY: Reperfusion injury: A review of the pathophysiology, clinical manifestations and therapeutic options. Int J Cardiol 58: 95-117, 1997.

3. Heusch G, Boengler K and Schulz R: Inhibition of mitochondrial permeability transition pore opening: The Holy Grail of cardioprotection. Basic Res Cardiol 105: 151-154, 2010.

4. GBD 2015 Disease and Injury Incidence and Prevalence Collaborators: Global, regional, and national incidence, prevalence, and years lived with disability for 310 diseases and injuries, 1990-2015: A systematic analysis for the Global Burden of Disease Study 2015. Lancet 388: 1545-1602, 2016.

5. Yellon DM and Hausenloy DJ: Myocardial reperfusion injury. N Engl J Med 357: 1121-1135, 2007.

6. Xiong J, Xue FS, Yuan YJ, Wang Q, Liao X and Wang WL: Cholinergic anti-inflammatory pathway: A possible approach to protect against myocardial ischemia reperfusion injury. Chin Med J (Engl) 123: 2720-2726, 2010.

7. Naidu BV, Farivar AS, Woolley SM, Grainger D, Verrier ED and Mulligan MS: Novel broad-spectrum chemokine inhibitor protects against lung ischemia-reperfusion injury. J Heart Lung Transplant 23: 128-134, 2004.

8. Olivetti G, Quaini F, Sala R, Lagrasta C, Corradi D, Bonacina E, Gambert SR, Cigola E and Anversa P: Acute myocardial infarction in humans is associated with activation of programmed myocyte cell death in the surviving portion of the heart. J Mol Cell Cardiol 28: 2005-2016, 1996.

9. Zhao ZQ, Nakamura M, Wang NP, Wilcox JN, Shearer S, Ronson RS, Guyton RA and Vinten-Johansen J: Reperfusion induces myocardial apoptotic cell death. Cardiovasc Res 45: 651-660, 2000

10. Orogo AM and Gustafsson AB: Cell death in the myocardium: My heart won't go on. IUBMB Life 65: 651-656, 2013.

11. Stephanou A, Brar B, Liao Z, Scarabelli T, Knight RA and Latchman DS: Distinct initiator caspases are required for the induction of apoptosis in cardiac myocytes during ischaemia versus reperfusion injury. Cell Death Differ 8: 434-435, 2001.

12. Scarabelli TM, Stephanou A, Pasini E, Comini L, Raddino R, Knight RA and Latchman DS: Different signaling pathways induce apoptosis in endothelial cells and cardiac myocytes during ischemia/reperfusion injury. Circ Res 90: 745-748, 2002.

13. Scarabelli T, Stephanou A, Rayment N, Pasini E, Comini L, Curello S, Ferrari R, Knight R and Latchman D: Apoptosis of endothelial cells precedes myocyte cell apoptosis in ischemia/reperfusion injury. Circulation 104: 253-256, 2001.

14. Jeremias I, Kupatt C, Martin-Villalba A, Habazettl H, Schenkel J, Boekstegers P and Debatin KM: Involvement of CD95/Apo1/Fas in cell death after myocardial ischemia. Circulation 102: 915-920, 2000.

15. Jin M, Li JR and Wu W: Study on the antioxidative effect of safflor yellow. Zhongguo Zhong Yao Za Zhi 29: 447-449, 2004 (In Chinese).

16. Wei X, Liu H, Sun X, Fu F, Zhang X, Wang J, An J and Ding H: Hydroxysafflor yellow A protects rat brains against ischemia-reperfusion injury by antioxidant action. Neurosci Lett 386: 58-62, 2005.

17. Darnell JE Jr: STATs and gene regulation. Science 277: $1630-1635,1997$.
18. Imada $\mathrm{K}$ and Leonard WJ: The Jak-STAT pathway. Mol Immunol 37: 1-11, 2000.

19. Negoro S, Kunisada K, Tone E, Funamoto M, Oh H, Kishimoto T and Yamauchi-Takihara K: Activation of JAK/STAT pathway transduces cytoprotective signal in rat acute myocardial infarction. Cardiovasc Res 47: 797-805, 2000.

20. Das A, Salloum FN, Durrant D, Ockaili R and Kukreja RC: Rapamycin protects against myocardial ischemia-reperfusion injury through JAK2-STAT3 signaling pathway. J Mol Cell Cardiol 53: 858-869, 2012.

21. Battle TE and Frank DA: The role of STATs in apoptosis. Curr Mol Med 2: 381-392, 2002.

22. Boengler K, Hilfiker-Kleiner D, Heusch G and Schulz R: Inhibition of permeability transition pore opening by mitochondrial STAT3 and its role in myocardial ischemia/reperfusion. Basic Res Cardiol 105: 771-785, 2010.

23. Heusch G, Musiolik J, Gedik N and Skyschally A: Mitochondrial STAT3 activation and cardioprotection by ischemic postconditioning in pigs with regional myocardial ischemia/reperfusion. Circ Res 109: 1302-1308, 2011

24. Yang N, Luo M, Li R, Huang Y, Zhang R, Wu Q, Wang F, Li Y and $\mathrm{Yu}$ X: Blockage of JAK/STAT signalling attenuates renal ischaemia-reperfusion injury in rat. Nephrol Dial Transplant 23: 91-100, 2008

25. Stephanou A, Brar BK, Knight RA and Latchman DS: Opposing actions of STAT- 1 and STAT- 3 on the Bcl- 2 and Bcl-x promoters. Cell Death Differ 7: 329-330, 2000.

26. Stephanou A, Scarabelli TM, Townsend PA, Bell R, Yellon D, Knight RA and Latchman DS: The carboxyl-terminal activation domain of the STAT-1 transcription factor enhances ischemia/reperfusion-induced apoptosis in cardiac myocytes. FASEB J 16: 1841-1843, 2002.

27. National Research Council (US) Committee for the Update of the Guide for the Care and Use of Laboratory Animals: Guide for the Care and Use of Laboratory Animals. 8th edition. National Academies Press (US), Washington, DC, 2011.

28. Zhu HB, Zhang L, Wang ZH, Tian JW, Fu FH, Liu K and Li CL: Therapeutic effects of hydroxysafflor yellow A on focal cerebral ischemic injury in rats and its primary mechanisms. J Asian Nat Prod Res 7: 607-613, 2005.

29. Liu SX, Zhang Y, Wang YF, Li XC, Xiang MX, Bian C and Chen P: Upregulation of heme oxygenase-1 expression by hydroxysafflor yellow A conferring protection from anoxia/reoxygenation-induced apoptosis in $\mathrm{H} 9 \mathrm{c} 2$ cardiomyocytes. Int $\mathrm{J}$ Cardiol 160: 95-101, 2012.

30. Wu YX, Gao CZ, Fan KL, Yang LM and Mei XF: STAT1 inhibitor alleviates spinal cord injury by decreasing apoptosis. Genet Mol Res 15: 2016.

31. Gorina R, Petegnief V, Chamorro A and Planas AM: AG490 prevents cell death after exposure of rat astrocytes to hydrogen peroxide or proinflammatory cytokines: Involvement of the Jak2/STAT pathway. J Neurochem 92: 505-518, 2005.

32. Smith CC, Dixon RA, Wynne AM, Theodorou L, Ong SG, Subrayan S, Davidson SM, Hausenloy DJ and Yellon DM: Leptin-induced cardioprotection involves JAK/STAT signaling that may be linked to the mitochondrial permeability transition pore. Am J Physiol Heart Circ Physiol 299: H1265-H1270, 2010.

33. Sachdeva J, Dai W, Gerczuk PZ and Kloner RA: Combined remote perconditioning and postconditioning failed to attenuate infarct size and contractile dysfunction in a rat model of coronary artery occlusion. J Cardiovasc Pharmacol Ther 19: 567-573, 2014.

34. Livak KJ and Schmittgen TD: Analysis of relative gene expression data using real-time quantitative PCR and the 2(-Delta Delta C(T)) method. Methods 25: 402-408, 2001.

35. Liu F, Wei Y, Yang XZ, Li FG, Hu J and Cheng RF: Hypotensive effects of safflower yellow in spontaneously hypertensive rats and influence on plasma renin activity and angiotensin II level. Yao Xue Xue Bao 27: 785-787, 1992 (In Chinese).

36. Ji DB, Zhang LY, Li CL, Ye J and Zhu HB: Effect of Hydroxysafflor yellow A on human umbilical vein endothelial cells under hypoxia. Vascul Pharmacol 50: 137-145, 2009.

37. Panteghini M, Cuccia C, Bonetti G, Giubbini R, Pagani F and Bonini E: Single-point cardiac troponin $\mathrm{T}$ at coronary care unit discharge after myocardial infarction correlates with infarct size and ejection fraction. Clin Chem 48: 1432-1436, 2002.

38. Kowalewski M, Urban M, Mroczko B and Szmitkowski M: Proinflammatory cytokines (IL-6, TNF-alpha) and cardiac troponin I (cTnI) in serum of young people with ventricular arrhythmias. Pol Arch Med Wewn 108: 647-651, 2002 (In Polish). 
39. Das DK, Engelman RM, Rousou JA, Breyer RH, Otani H and Lemeshow S: Pathophysiology of superoxide radical as potential mediator of reperfusion injury in pig heart. Basic Res Cardiol 81: $155-166,1986$.

40. Wu J, Hecker JG and Chiamvimonvat N: Antioxidant enzyme gene transfer for ischemic diseases. Adv Drug Deliv Rev 61: 351-363, 2009.

41. Taiwo T and Goldstein S: Drug use and its association with deviant behaviour among rural adolescent students in South Africa. East Afr Med J 83: 500-506, 2006.

42. Scarabelli TM, Knight R, Stephanou A, Townsend P, Chen-Scarabelli C, Lawrence K, Gottlieb R, Latchman D and Narula J: Clinical implications of apoptosis in ischemic myocardium. Curr Probl Cardiol 31: 181-264, 2006.

43. Salvesen GS: Caspases and apoptosis. Essays Biochem 38: 9-19, 2002.

44. Barry SP, Townsend PA, Latchman DS and Stephanou A: Role of the JAK-STAT pathway in myocardial injury. Trends Mol Med 13: 82-89, 2007.

45. Shinmura K, Tang XL, Wang Y, Xuan YT, Liu SQ, Takano H, Bhatnagar A and Bolli R: Cyclooxygenase-2 mediates the cardioprotective effects of the late phase of ischemic preconditioning in conscious rabbits. Proc Natl Acad Sci USA 97: 10197-10202, 2000.

46. Fuglesteg BN, Suleman N, Tiron C, Kanhema T, Lacerda L, Andreasen TV, Sack MN, Jonassen AK, Mjøs OD, Opie LH and Lecour S: Signal transducer and activator of transcription 3 is involved in the cardioprotective signalling pathway activated by insulin therapy at reperfusion. Basic Res Cardiol 103: 444-453, 2008.

47. Mascareno E, El-Shafei M, Maulik N, Sato M, Guo Y, Das DK and Siddiqui MA: JAK/STAT signaling is associated with cardiac dysfunction during ischemia and reperfusion. Circulation 104: 325-329, 2001

48. Simon AR, Rai U, Fanburg BL and Cochran BH: Activation of the JAK-STAT pathway by reactive oxygen species. Am J Physiol 275: C1640-C1652, 1998.

49. Takagi Y, Harada J, Chiarugi A and Moskowitz MA: STAT1 is activated in neurons after ischemia and contributes to ischemic brain injury. J Cereb Blood Flow Metab 22: 1311-1318, 2002.

50. Sinha K, Das J, Pal PB and Sil PC: Oxidative stress: The mitochondria-dependent and mitochondria-independent pathways of apoptosis. Arch Toxicol 87: 1157-1180, 2013.

51. Wang J, Ouyang C, Chen X, Fu B, Lu Y and Hong Q: Effect of Jak2 kinase inhibition on Stat 1 and Stat 3 activation and apoptosis of tubular epithelial cells induced by ATP depletion/recovery. J Nephrol 21: 919-923, 2008.
52. Dhingra S, Bagchi AK, Ludke AL, Sharma AK and Singal PK: Akt regulates IL-10 mediated suppression of TNFa-induced cardiomyocyte apoptosis by upregulating Stat 3 phosphorylation. PLoS One 6: e25009, 2011.

53. Stephanou A, Brar BK, Scarabelli TM, Jonassen AK, Yellon DM, Marber MS, Knight RA and Latchman DS: Ischemia-induced STAT-1 expression and activation play a critical role in cardiomyocyte apoptosis. J Biol Chem 275: 10002-10008, 2000.

54. Bolli R, Dawn B and Xuan YT: Role of the JAK-STAT pathway in protection against myocardial ischemia/reperfusion injury. Trends Cardiovasc Med 13: 72-79, 2003

55. Sheng M, Huang Z, Pan L, Yu M, Yi C, Teng L, He L, Gu C, $\mathrm{Xu} \mathrm{C}$ and Li J: SOCS2 exacerbates myocardial injury induced by ischemia/reperfusion in diabetic mice and $\mathrm{H} 9 \mathrm{c} 2$ cells through inhibiting the JAK-STAT-IGF-1 pathway. Life Sci 188: 101-109, 2017.

56. Yamaura G, Turoczi T, Yamamoto F, Siddqui MA, Maulik N and Das DK: STAT signaling in ischemic heart: A role of STAT5A in ischemic preconditioning. Am J Physiol Heart Circ Physiol 285: H476-H482, 2003.

57. Jung YY, Lee JH, Nam D, Narula AS, Namjoshi OA, Blough BE, Um JY, Sethi G and Ahn KS: Anti-myeloma effects of icariin are mediated through the attenuation of JAK/STAT3-dependent signaling cascade. Front Pharmacol 9: 531, 2018.

58. Han D, Wei J, Zhang R, Ma W, Shen C, Feng Y, Xia N, Xu D, Cai D, Li Y and Fang W: Hydroxysafflor yellow A alleviates myocardial ischemia/reperfusion in hyperlipidemic animals through the suppression of TLR4 signaling. Sci Rep 6: 35319 , 2016.

59. Min J and Wei C: Hydroxysafflor yellow A cardioprotection in ischemia-reperfusion (I/R) injury mainly via Akt/hexokinase II independent of ERK/GSK-3 $\beta$ pathway. Biomed Pharmacother 87: 419-426, 2017.

60. Xu AB, Liu JG, Zhang J and LI JX: Hydroxysafflor yellow A for ameliorating injury of myocardial ischemia/reperfusion in SD rats. Chin J Evidence Based Cardiovascular Med 6: 733-736, 2014 (In Chinese).

This work is licensed under a Creative Commons Attribution-NonCommercial-NoDerivatives 4.0 International (CC BY-NC-ND 4.0) License. 